\title{
Some results on discrete eigenvalues for the Stochastic Nonlinear Schrödinger Equation in fiber optics
}

\author{
Laura Prati ${ }^{1}$, Luigi Barletti ${ }^{1 *}$ \\ ${ }^{1}$ Dipartimento di Matematica e Informatica "U.Dini", \\ Università degli Studi di Firenze, Italy \\ *Email address for correspondence: luigi.barletti@unifi.it \\ Communicated by Renato Spigler \\ Received on 01 30, 2017. Accepted on 20 20, 2018.
}

\begin{abstract}
We study a stochastic Nonlinear Schrödinger Equation (NLSE), with additive white Gaussian noise, by means of the Nonlinear Fourier Transform (NFT). In particular, we focus on the propagation of discrete eigenvalues along a focusing fiber. Since the stochastic NLSE is not exactly integrable by means of the NFT, then we use a perturbation approach, where we assume that the signal-to-noise ratio is high. The zeroth-order perturbation leads to the deterministic NLSE while the first-order perturbation allows to describe the statistics of the discrete eigenvalues. This is important to understand the properties of the channel for recently devised optical transmission techniques, where the information is encoded in the nonlinear Fourier spectrum.

Keywords: Stochastic Nonlinear Schrödinger Equation, Nonlinear Fourier Transform, Eigenvalues, Solitons, Perturbation, Fiber Optics.

AMS subject classification: 35Q60, 81U15, 37K15, 35R60.
\end{abstract}

\section{Introduction.}

The Nonlinear Schrödinger Equation (NLSE) governs the evolution of the complex envelope of signals propagating in optical fibers [1,2]. The stochastic version of the NLSE also introduces the presence of optical noise, which is added to non-linear effects of propagation along the fiber. For a single-mode fiber, the stochastic NLSE in case of anomalous dispersion (focusing fiber) is

$$
\frac{\partial q}{\partial z}(z, t)=\frac{i}{2} \frac{\partial^{2} q}{\partial t^{2}}(z, t)+i q(z, t)|q(z, t)|^{2}+\eta(z, t)
$$


where $q(z, t)$ is the complex field envelope, $z \in \mathbb{R}^{+}$is the normalized distance along the fiber, $t \in \mathbb{R}$ is time in a frame moving with the group velocity of the envelope, and $\eta(z, t)$ is the additive white Gaussian noise having zero mean [3]:

$$
\langle\eta(z, t)\rangle=0 .
$$

Let $\eta_{1}$ and $\eta_{2}$ denote the real and imaginary parts, respectively, of noise:

$$
\eta(z, t)=\eta_{1}(z, t)+i \eta_{2}(z, t) .
$$

We assume

$$
\left\langle\eta_{i}(z, t) \eta_{j}\left(z^{\prime}, t^{\prime}\right)\right\rangle=D \delta\left(t-t^{\prime}\right) \delta\left(z-z^{\prime}\right) \delta_{i j}, \quad i, j \in\{1,2\}
$$

where $D>0$ is the spectral density of noise [4]. If the superscript $*$ represents complex conjugation, we have

$$
\left\langle\eta(z, t) \eta^{*}\left(z^{\prime}, t^{\prime}\right)\right\rangle=\left\langle\eta^{*}(z, t) \eta\left(z^{\prime}, t^{\prime}\right)\right\rangle=2 D \delta\left(t-t^{\prime}\right) \delta\left(z-z^{\prime}\right)
$$

and

$$
\left\langle\eta(z, t) \eta\left(z^{\prime}, t^{\prime}\right)\right\rangle=\left\langle\eta^{*}(z, t) \eta^{*}\left(z^{\prime}, t^{\prime}\right)\right\rangle=0 .
$$

The stochastic NLSE (1) models the chromatic dispersion (first term in the right hand side), the Kerr nonlinearity (second term in the right hand side) and noise: these are physical aspects which seriously affect system performance during the propagation of the optical signal [1,2].

Equation (1) has not been proven to be well-posed and is likely illposed [5]. Nevertheless, it is widely used in the physical and engineering literature $[1,6-8]$. The problem can be mathematically fixed just with a cutoff in the spectrum of noise [9]. We do not introduce such a cutoff for simplicity of tractation.

The optical signal travels along the fiber moving from the transmitter to the receiver, which is possibly located tens of kilometers away. It implies the risk of a strong distortion of the signal, making it difficult to decode the information at the receiver and limiting the transmission rate of the information itself.

Current optical networks mostly employ methodologies originally developed for linear channels, and nonlinearity has a detrimental effect on this kind of transmission. In particular, it has been estimated that the existing optical fiber technology is going to approach the so called nonlinear transmission limit [10-15]. Linearization approaches to the NLSE can be found in [2] and references therein and in [16]. 


\section{Discrete eigenvalues for the Stochastic Nonlinear Schrödinger Equation}

Nowadays, part of the scientific community's efforts is devoted to develop technology for nonlinear and noisy transmissions.

In this work, we approach the issue of nonlinearity by means of the Nonlinear Fourier Transform (NFT) [6,17], which is a powerful tool to treat the (by definition) integrable nonlinear partial differential equations, like the deterministic NLSE [18-21]:

$$
\frac{\partial q}{\partial z}(z, t)=\frac{i}{2} \frac{\partial^{2} q}{\partial t^{2}}(z, t)+i q(z, t)|q(z, t)|^{2} .
$$

The NFT maps the optical signal (i.e. the solution of the NLSE) onto a set of scattering data -constituted by the continuous spectral function, the discrete eigenvalues and the discrete spectral function associated to the deterministic NLSE (7)- which evolve in a trivial manner along the fiber. On this ground, in [17] it was proposed to encode and transmit the information not on the optical signal itself, affected by dispersion and nonlinearity, but on the discrete eigenvalues associated to it, because they evolve linearly. This approach was named eigenvalue communication. The choice of using the discrete spectrum for encoding the information has been explored by several authors, see [22-27]. Also the possibility of using the continuous spectrum has been approached, see [8,28-31], often under the name of nonlinear inverse synthesis.

We focus on the behavior of the discrete eigenvalues during the propagation along the fiber in the presence of additive white Gaussian noise in order to understand and describe their statistics and, as a direct consequence, the properties of the channel. Following $[8,25]$, we use a perturbation approach. Deterministic perturbation analysis for discrete eigenvalues can be found in [32-36], but a stochastic distribution of the spectral data is not faced. In [37] the special case of a noise-free channel with a white Gaussian stochastic input is studied and the distributions of the spectral data are computed. In many works the statistics for the single-soliton case are studied, see [38] and references therein. In [25] the perturbation of discrete eigenvalues is studied in order to simulate their statistics on a computer; the first-order variation of eigenvalues is obtained and analytical computations for the statistics are given explicitly for the one-soliton case.

In this work we examine in depth the non deterministic terms because the comprehension of their statistics lays the foundation for understanding the influence of noise on the received discrete spectrum. We assume that the signal-to-noise ratio is high ( $\eta$ small) and we follow the perturbative approach of $[8,25]$ to study analytically the statistics, in order to determine the hierarchy of perturbation equations for the propagation of eigenvalues. The zero-order perturbation leads to the deterministic NLSE (7). 
In Section 2.1 we briefly recall the NFT for the deterministic NLSE (7) and its notation. In Section 2.2 we recall the evolution equations for the scattering data, also for small perturbations in the NFT. In Section 3 we compute the output for discrete eigenvalues at the end of the fiber and the related statistics. As an example, in Section 3.2 we make explicit computations in the one-soliton case. In Section 4 we summarize some brief conclusions.

\section{Notation.}

\subsection{Basics on the Nonlinear Fourier Transform.}

The Nonlinear Fourier Transform (NFT) is the forward step of the Inverse Scattering Transform (IST) technique, which was developed in the 1960s and later for solving certain nonlinear partial differential equations (PDEs), called integrable [18,19,39-42], of the form

$$
q_{z}=K(q)
$$

where $q=q(z, t), z \geq 0, t \in \mathbb{R}$, is sufficiently smooth and vanishing as $t \rightarrow \pm \infty$ together with all its derivatives, and $K$ is a differential operator acting on $q(z, \cdot)$. Numerical approaches to treat the NFT can be seen in $[7,43,44]$ where fast algorithms are described.

If $q$ is the solution of the deterministic NLSE (7), which is integrable, one sets the so called Zakharov-Shabat (ZS) system for the evolution of the scattering data as:

$$
\left\{\begin{array}{l}
v_{1 t}(z, t)+i \zeta v_{1}(z, t)=q(z, t) v_{2}(z, t) \\
v_{2 t}(z, t)-i \zeta v_{2}(z, t)=-q^{*}(z, t) v_{1}(z, t)
\end{array}\right.
$$

where $t \in \mathbb{R}$ is time, $z \in[0, \mathcal{L}]$ is the distance along a fiber of length $\mathcal{L}$, $v(z, t)=\left(v_{1}(z, t), v_{2}(z, t)\right)$ are the eigenfunctions, $\zeta \in \mathbb{C}$ are the eigenvalues, and $q^{*}(z, t)$ is the complex conjugate of $q(z, t)$, that plays the role of a potential in the ZS system (9). We assume that $q$ satisfies

$$
\int_{-\infty}^{+\infty}|q(z, t)| d t<\infty
$$

and

$$
q(z, t) \rightarrow 0 \quad \text { for }|t| \rightarrow \infty .
$$

The eigenvalues $\zeta$ are generally complex:

$$
\zeta=\xi+i \mu \in \mathbb{C} \quad \text { with } \xi, \mu \in \mathbb{R} ;
$$




\section{Discrete eigenvalues for the Stochastic Nonlinear Schrödinger Equation}

when an eigenvalue is real, it is represented by just $\xi \in \mathbb{R}$ :

$$
\zeta=\xi \in \mathbb{R} .
$$

Let us consider specific eigenfunctions $\phi, \bar{\phi}, \psi, \bar{\psi}$ of the ZS system (9) called Jost functions (overbar does not mean complex conjugation) with the following asymptotic behavior for $|t| \rightarrow \infty$ :

$$
\begin{aligned}
& \phi(z, t ; \xi)=\left(\begin{array}{c}
\phi_{1}(z, t ; \xi) \\
\phi_{2}(z, t ; \xi)
\end{array}\right) \rightarrow\left(\begin{array}{c}
\mathrm{e}^{-i \xi t} \\
0
\end{array}\right) \quad \text { as } t \rightarrow-\infty \\
& \bar{\phi}(z, t ; \xi)=\left(\begin{array}{c}
\bar{\phi}_{1}(z, t ; \xi) \\
\bar{\phi}_{2}(z, t ; \xi)
\end{array}\right) \rightarrow\left(\begin{array}{c}
0 \\
-\mathrm{e}^{i \xi t}
\end{array}\right) \quad \text { as } t \rightarrow-\infty \\
& \psi(z, t ; \xi)=\left(\begin{array}{l}
\psi_{1}(z, t ; \xi) \\
\psi_{2}(z, t ; \xi)
\end{array}\right) \rightarrow\left(\begin{array}{c}
0 \\
e^{i \xi t}
\end{array}\right) \quad \text { as } t \rightarrow+\infty \\
& \bar{\psi}(z, t ; \xi)=\left(\begin{array}{l}
\bar{\psi}_{1}(z, t ; \xi) \\
\bar{\psi}_{2}(z, t ; \xi)
\end{array}\right) \rightarrow\left(\begin{array}{c}
\mathrm{e}^{-i \xi t} \\
0
\end{array}\right) \quad \text { as } t \rightarrow+\infty
\end{aligned}
$$

The right and left solutions are related by the Jost coefficients $a, b, \bar{a}, \bar{b}$

$$
\begin{aligned}
& \psi(z, t ; \xi)=-a(\xi, z) \bar{\phi}(z, t ; \xi)+\bar{b}(\xi, z) \phi(z, t ; \xi) \\
& \bar{\psi}(z, t ; \xi)=\bar{a}(\xi, z) \phi(z, t ; \xi)+b(\xi, z) \bar{\phi}(z, t ; \xi)
\end{aligned}
$$

where

$$
a(\xi, z) \bar{a}(\xi, z)+b(\xi, z) \bar{b}(\xi, z)=1 .
$$

Functions $a, \bar{a}$ can be analytically extended (in $\xi$ ) into the upper and lower half complex plane respectively [19]. Therefore, in addition to the continuous spectrum $(\xi \in \mathbb{R})$, there is also the discrete spectrum, that occurs for $\zeta_{k} \in \mathbb{C}^{+}$such that

$$
a\left(\zeta_{k}, z\right)=0 \quad k=1,2, \ldots, N
$$

( $N$ is finite, see [19]). If $\zeta_{k} \in \mathbb{C}^{+}$is an eigenvalue, also $\zeta_{k}^{*} \in \mathbb{C}^{-}$is a discrete eigenvalue in the lower half plane, therefore one omits to consider the zeros of $\bar{a}$ and limits the study of discrete eigenvalues to the upper half plane. The Jost functions $\phi, \psi$ become linearly dependent [19]:

$$
\phi\left(z, t ; \zeta_{k}\right)=b\left(\zeta_{k}, z\right) \psi\left(z, t ; \zeta_{k}\right) \quad \text { for } k=1,2, \ldots, N .
$$

The asymptotic behavior of the Jost functions for discrete eigenvalues $\zeta_{k} \in \mathbb{C}^{+}$is given by

$$
\begin{aligned}
& \phi\left(z, t ; \zeta_{k}\right)=\left(\begin{array}{l}
\phi_{1}\left(z, t ; \zeta_{k}\right) \\
\phi_{2}\left(z, t ; \zeta_{k}\right)
\end{array}\right) \rightarrow\left(\begin{array}{c}
\mathrm{e}^{-i \zeta_{k} t} \\
0
\end{array}\right) \quad \text { as } t \rightarrow-\infty \\
& \psi\left(z, t ; \zeta_{k}\right)=\left(\begin{array}{l}
\psi_{1}\left(z, t ; \zeta_{k}\right) \\
\psi_{2}\left(z, t ; \zeta_{k}\right)
\end{array}\right) \rightarrow\left(\begin{array}{c}
0 \\
\mathrm{e}^{i \zeta_{k} t}
\end{array}\right) \quad \text { as } t \rightarrow+\infty
\end{aligned}
$$




\section{Prati, L. Barletti}

Definition 2.1. The set of scattering data is given by

$$
S_{+}:=\{\rho(\xi), \xi \in \mathbb{R}\} \cup\left\{\zeta_{k} \in \mathbb{C}^{+}, C_{k}\right\}_{k=1}^{N}
$$

where

$$
\rho(\xi, z):=\frac{b(\xi, z)}{a(\xi, z)}, \quad \text { for } \xi \in \mathbb{R},
$$

is called the continuous spectral function, defined on the continuous spectrum $\xi \in \mathbb{R}, \zeta_{k}$ are the discrete eigenvalues in the upper half plane $\mathbb{C}^{+}$, and

$$
C_{k}:=\frac{b\left(\zeta_{k}, z\right)}{a_{\zeta}\left(\zeta_{k}, z\right)}, \quad \text { for } k=1,2, \ldots, N
$$

is called the discrete spectral function.

The NFT maps the nonlinear PDE one aims at resolving into its set of scattering data.

\subsection{Evolution of the scattering data and perturbations.}

In order to give the evolution equations for the scattering data, we are going to use the compact notation of [32].

Definition 2.2. If $q(z, t)$ satisfies an evolution equation

$$
q_{z}=K(q)
$$

with $K$ nonlinear operator acting on $q$ as a function of $t$, the Kaup operator $I[u, v ; \zeta]$ acts on $u=\left(u_{1}, u_{2}\right), v=\left(v_{1}, v_{2}\right)$ like

$$
I[u, v ; \zeta]:=\int_{-\infty}^{+\infty}\left[i q_{z}(z, t) u_{2}(z, t ; \zeta) v_{2}(z, t ; \zeta)+i q_{z}^{*}(z, t) u_{1}(z, t ; \zeta) v_{1}(z, t ; \zeta)\right] d t .
$$

Notice that the operator $I$ is bilinear. With this notation, the evolution of scattering coefficients $a, b$ and scattering data $\rho, \zeta_{k}$ is given by [32]

$$
\begin{aligned}
i a_{z}(\zeta, z) & =I[\phi, \psi ; \zeta], \\
i b_{z}(\zeta, z) & =-I[\phi, \bar{\psi} ; \zeta], \\
i \rho_{z}(\xi, z) & =-\frac{1}{a^{2}(\xi, z)} I[\phi, \phi ; \xi], \\
i \frac{d}{d z} \zeta_{k}(z) & =-C_{k} I\left[\psi, \psi ; \zeta_{k}\right], \quad \text { for } k=1,2, \ldots, N,
\end{aligned}
$$




\section{Discrete eigenvalues for the Stochastic Nonlinear Schrödinger Equation}

where $\phi, \psi$ and $\bar{\psi}$ are Jost functions of the ZS system associated to $K$ (we omit the complicated evolution of $C_{k}$ because not useful here). When $q$ satisfies the deterministic NLSE (7), one uses index 0 for the Kaup operator $I_{0}[u, v ; \zeta]$ and the evolution equations simplify to:

$$
\begin{aligned}
i a_{z}(\zeta, z) & =0, \\
i b_{z}(\zeta, z) & =-2 \zeta^{2} b(\zeta, z), \\
i \rho_{z}(\xi, z) & =-2 \xi^{2} \rho(\xi, z), \\
i \frac{d}{d z} \zeta_{k}(z) & =0, \quad \text { for } k=1, \ldots, N, \\
i C_{k, z}(z) & =-2 \zeta_{k}^{2} C_{k}(z), \quad \text { for } k=1, \ldots, N .
\end{aligned}
$$

The evolution is trivial and the number of the discrete eigenvalues, which are zeros of $a$, is preserved.

In $[32,34]$ a perturbation theory is developed for $q$ satisfying

$$
q_{z}(z, t)=K_{0}(q)+\sigma K_{1}(q),
$$

with $K_{0}$ nonlinear operator for an exactly integrable case, like the deterministic NLSE (7), $K_{1}$ operator for the perturbation and $\sigma$ a small parameter. Briefly, for the perturbation of the deterministic NLSE (7), one has

$$
I[u, v ; \zeta]=I_{0}[u, v ; \zeta]+\sigma I_{1}[u, v ; \zeta],
$$

where

$$
\begin{aligned}
& I_{1}[u, v ; \zeta]:= \\
& \quad \int_{-\infty}^{+\infty}\left[i K_{1}(q) u_{2}(z, t ; \zeta) v_{2}(z, t ; \zeta)+i\left[K_{1}(q)\right]^{*} u_{1}(z, t ; \zeta) v_{1}(z, t ; \zeta)\right] d t
\end{aligned}
$$

should be understood as computed for $u, v$ in the unperturbed problem (zero-order perturbation), therefore known (full information).

This framework can therefore be used in order to treat the stochastic NLSE (1) for the propagation of signal along the fiber [8] by interpreting $K_{1}$ as the additive noise term.

\section{Propagation of discrete eigenvalues.}

Following $[8,25]$, we consider a small noise $\eta$, compared with the signal power, and apply the NFT perturbation theory to obtain a stochastic description of the channel. In [8] the stochastic evolution for the continuous spectral function is studied. We are going to study the evolution for 
the discrete eigenvalues following Kaup's notation. A similar perturbation approach to obtain the evolution equation for discrete eigenvalues is given in [25], where the perturbation of discrete eigenvalues is studied in order to simulate their statistics on a computer and analytical computations are given explicitly only for the one-soliton case. By means of the Kaup notation, we not only solve the evolution equation for discrete eigenvalues, but we are also able (in principle) to give a complete description of the statistics of the channel for the multi-solitonic case, corresponding to an arbitrary number $N$ of eigenvalues in the upper half complex plane.

From (28) we have that the equation for the evolution of the discrete eigenvalues $\zeta_{k}=\zeta_{k}(z)$, for $k=1,2, \ldots, N$ is therefore given by:

$$
i \frac{d}{d z} \zeta_{k}(z)=-C_{k}(z) I_{1}^{\text {noise }}\left[\psi, \psi ; \zeta_{k}\right]
$$

where $C_{k}(z)$ is defined in Equation (22) and, according to (36),

$$
\begin{aligned}
& I_{1}^{\text {noise }}[u, v ; \zeta]:= \\
& \quad \int_{-\infty}^{+\infty}\left[i \eta(z, t) u_{2}(z, t ; \zeta) v_{2}(z, t ; \zeta)+i \eta^{*}(z, t) u_{1}(z, t ; \zeta) v_{1}(z, t ; \zeta)\right] d t
\end{aligned}
$$

Equation (37) can be written more explicitly as

$$
\frac{d}{d z} \zeta_{k}(z)=-C_{k}(z) \int_{-\infty}^{+\infty}\left[\eta(z, t) \psi_{2}^{2}\left(z, t ; \zeta_{k}\right)+\eta^{*}(z, t) \psi_{1}^{2}\left(z, t ; \zeta_{k}\right)\right] d t
$$

or, concisely,

$$
\frac{d}{d z} \zeta_{k}(z)=\Delta\left(\zeta_{k}, z\right)
$$

where

$$
\Delta\left(\zeta_{k}, z\right):=-C_{k}(z) \int_{-\infty}^{+\infty}\left[\eta(z, t) \psi_{2}^{2}\left(z, t ; \zeta_{k}\right)+\eta^{*}(z, t) \psi_{1}^{2}\left(z, t ; \zeta_{k}\right)\right] d t
$$

Note, moreover, that

$$
C_{k}(z)=C_{k}\left(\zeta_{k}(0), z\right)=\frac{b\left(\zeta=\zeta_{k}(0), z\right)}{a_{\zeta}\left(\zeta=\zeta_{k}(0), z\right)}
$$

since the Jost functions and the Jost coefficients should be understood as those of the unperturbed problem and are assumed to be known from the leading order. 


\section{Discrete eigenvalues for the Stochastic Nonlinear Schrödinger Equation}

Let us compute the statistics of noise $\Delta\left(\zeta_{k}, z\right)$ to completely determine it. The mean is null:

$$
\left\langle\Delta\left(\zeta_{k}, z\right)\right\rangle=0
$$

because $\langle\eta(z, t)\rangle=0$, see Equation (2). This implies that

$$
\frac{d}{d z}\left\langle\zeta_{k}(z)\right\rangle=0
$$

and then, from Equations (40) and (43),

$$
\left\langle\zeta_{k}(z)\right\rangle=\left\langle\zeta_{k}(0)\right\rangle=\zeta_{k}(0) .
$$

Hence, the mean value of each discrete eigenvalue is constant and equals its value at $z=0$.

The autocorrelations to be evaluated are $\left\langle\Delta\left(\zeta_{k}, z\right) \Delta^{*}\left(\zeta_{j}, z^{\prime}\right)\right\rangle$ and $\left\langle\Delta\left(\zeta_{k}, z\right) \Delta\left(\zeta_{j}, z^{\prime}\right)\right\rangle$ for $\zeta_{k} \neq \zeta_{j}, z \neq z^{\prime}$. For the first one, one obtains

$$
\begin{aligned}
&\left\langle\Delta\left(\zeta_{k}, z\right) \Delta^{*}\left(\zeta_{j}, z^{\prime}\right)\right\rangle=C_{k}(z) C_{j}^{*}\left(z^{\prime}\right) \times \\
& \times \int_{-\infty}^{+\infty} d t \int_{-\infty}^{+\infty} d t^{\prime} {\left[2 D \delta\left(t-t^{\prime}\right) \delta\left(z-z^{\prime}\right) \psi_{2}^{2}\left(z, t ; \zeta_{k}\right) \psi_{2}^{* 2}\left(z^{\prime}, t^{\prime} ; \zeta_{j}\right)+\right.} \\
&\left.+2 D \delta\left(t-t^{\prime}\right) \delta\left(z-z^{\prime}\right) \psi_{1}^{2}\left(z, t ; \zeta_{k}\right) \psi_{1}^{* 2}\left(z^{\prime}, t^{\prime} ; \zeta_{j}\right)\right],
\end{aligned}
$$

where $D>0$ is the spectral density of noise, see Equation (4); hence

$$
\begin{aligned}
& \left\langle\Delta\left(\zeta_{k}, z\right) \Delta^{*}\left(\zeta_{j}, z^{\prime}\right)\right\rangle=C_{k}(z) C_{j}^{*}\left(z^{\prime}\right) 2 D \delta\left(z-z^{\prime}\right) \times \\
& \quad \times \int_{-\infty}^{+\infty}\left[\psi_{2}^{2}\left(z, t ; \zeta_{k}\right) \psi_{2}^{* 2}\left(z^{\prime}, t ; \zeta_{j}\right)+\psi_{1}^{2}\left(z, t ; \zeta_{k}\right) \psi_{1}^{* 2}\left(z^{\prime}, t ; \zeta_{j}\right)\right] d t,
\end{aligned}
$$

and eventually, because of the presence of the term $\delta\left(z-z^{\prime}\right)$

$$
\begin{aligned}
& \left\langle\Delta\left(\zeta_{k}, z\right) \Delta^{*}\left(\zeta_{j}, z^{\prime}\right)\right\rangle=C_{k}(z) C_{j}^{*}(z) 2 D \delta\left(z-z^{\prime}\right) \times \\
& \quad \times \int_{-\infty}^{+\infty}\left[\psi_{2}^{2}\left(z, t ; \zeta_{k}\right) \psi_{2}^{* 2}\left(z, t ; \zeta_{j}\right)+\psi_{1}^{2}\left(z, t ; \zeta_{k}\right) \psi_{1}^{* 2}\left(z, t ; \zeta_{j}\right)\right] d t .
\end{aligned}
$$

Briefly, it is

$$
\left\langle\Delta\left(\zeta_{k}, z\right) \Delta^{*}\left(\zeta_{j}, z^{\prime}\right)\right\rangle=2 D C\left(z ; \zeta_{k}, \zeta_{j}\right) \delta\left(z-z^{\prime}\right)
$$

where

$$
\begin{aligned}
& C\left(z ; \zeta_{k}, \zeta_{j}\right):=C_{k}(z) C_{j}^{*}(z) \times \\
& \quad \times \int_{-\infty}^{+\infty}\left[\psi_{2}^{2}\left(z, t ; \zeta_{k}\right) \psi_{2}^{* 2}\left(z, t ; \zeta_{j}\right)+\psi_{1}^{2}\left(z, t ; \zeta_{k}\right) \psi_{1}^{* 2}\left(z, t ; \zeta_{j}\right)\right] d t
\end{aligned}
$$




\section{Prati, L. Barletti}

Similarly

$$
\left\langle\Delta\left(\zeta_{k}, z\right) \Delta\left(\zeta_{j}, z^{\prime}\right)\right\rangle=2 D E\left(z ; \zeta_{k}, \zeta_{j}\right) \delta\left(z-z^{\prime}\right)
$$

where

$$
\begin{aligned}
E\left(z ; \zeta_{k}, \zeta_{j}\right) & :=C_{k}(z) C_{j}(z) \times \\
& \times \int_{-\infty}^{+\infty}\left[\psi_{2}^{2}\left(z, t ; \zeta_{k}\right) \psi_{1}^{2}\left(z, t ; \zeta_{j}\right)+\psi_{1}^{2}\left(z, t ; \zeta_{k}\right) \psi_{2}^{2}\left(z, t ; \zeta_{j}\right)\right] d t
\end{aligned}
$$

The autocorrelation functions given in Equations (47) and (49) do make sense, because integrals in Equations (48) and (50) both converge because of the decay properties of the Jost functions (19). Indeed, the integrands are continuous functions and they decay exponentially where the interval of integration goes to infinity. For example we show the convergence of the integral defining $C\left(z ; \zeta_{k}, \zeta_{j}\right)$ in Equation (48). Consider an arbitrary $T>0$. Then we have:

$$
\begin{gathered}
C_{k}(z) C_{j}^{*}(z) \int_{-\infty}^{-\frac{T}{2}}\left[\psi_{2}^{2}\left(z, t ; \zeta_{k}\right) \psi_{2}^{* 2}\left(z, t ; \zeta_{j}\right)+\psi_{1}^{2}\left(z, t ; \zeta_{k}\right) \psi_{1}^{* 2}\left(z, t ; \zeta_{j}\right)\right] d t \\
=C_{k}(z) C_{j}^{*}(z) b^{-2}\left(\zeta_{k}, z\right) b^{*-2}\left(\zeta_{j}, z\right) \int_{-\infty}^{-\frac{T}{2}} \mathrm{e}^{-2 i\left(\zeta_{k}-\zeta_{j}^{*}\right) t} d t+O\left(\frac{1}{T}\right) \\
=-\frac{C_{k}(z) C_{j}^{*}(z)}{b^{2}\left(\zeta_{k}, z\right) b^{* 2}\left(\zeta_{j}, z\right)} \frac{1}{2 i\left(\zeta_{k}-\zeta_{j}^{*}\right)} \mathrm{e}^{i\left(\zeta_{k}-\zeta_{j}^{*}\right) T}+O\left(\frac{1}{T}\right)
\end{gathered}
$$

where $\zeta_{k}=\alpha_{k}+i \beta_{k}$ with $\beta_{k} \in \mathbb{R}^{+}$and $\zeta_{j}=\alpha_{j}+i \beta_{j}$ with $\beta_{j} \in \mathbb{R}^{+}$, then $i\left(\zeta_{k}-\zeta_{j}^{*}\right)$ has a negative real part. Similarly, on the right we have:

$$
\begin{array}{r}
C_{k}(z) C_{j}^{*}(z) \int_{\frac{T}{2}}^{+\infty}\left[\psi_{2}^{2}\left(z, t ; \zeta_{k}\right) \psi_{2}^{* 2}\left(z, t ; \zeta_{j}\right)++\psi_{1}^{2}\left(z, t ; \zeta_{k}\right) \psi_{1}^{* 2}\left(z, t ; \zeta_{j}\right)\right] d t \\
=-C_{k}(z) C_{j}^{*}(z) \frac{1}{2 i\left(\zeta_{k}-\zeta_{j}^{*}\right)} \mathrm{e}^{i\left(\zeta_{k}-\zeta_{j}^{*}\right) T}+O\left(\frac{1}{T}\right)
\end{array}
$$

The convergence of $E\left(z ; \zeta_{k}, \zeta_{j}\right)$ can be shown easily in a similar way.

\subsection{The channel output.}

Let now $\zeta_{k}(\mathcal{L})$ be the channel output, defined as the solution of Eq.(40) at the receiver located at distance $z=\mathcal{L}$. Equation (40) gives

$$
\zeta_{k}(z)=\zeta_{k}(0)+\int_{0}^{z} \Delta\left(\zeta_{k}(0), z^{\prime}\right) d z^{\prime}
$$




\section{Discrete eigenvalues for the Stochastic Nonlinear Schrödinger Equation}

therefore the channel output is

$$
\zeta_{k}(\mathcal{L})=\zeta_{k}(0)+\int_{0}^{\mathcal{L}} \Delta\left(\zeta_{k}(0), z\right) d z
$$

or shortly

$$
\zeta_{k}(\mathcal{L})=\zeta_{k}(0)+N\left(\zeta_{k}\right)
$$

where

$$
N\left(\zeta_{k}\right):=\int_{0}^{\mathcal{L}} \Delta\left(\zeta_{k}, z\right) d z .
$$

Let us compute the statistics of $N\left(\zeta_{k}\right)$. The mean is

$$
\left\langle N\left(\zeta_{k}\right)\right\rangle=0
$$

because of Equation (43). Then we compute the two autocorrelations $\left\langle N\left(\zeta_{k}\right) N^{*}\left(\zeta_{j}\right)\right\rangle$ and $\left\langle N\left(\zeta_{k}\right) N\left(\zeta_{j}\right)\right\rangle$ for $\zeta_{k} \neq \zeta_{j}$. The first one is

$$
\begin{aligned}
\left\langle N\left(\zeta_{k}\right) N^{*}\left(\zeta_{j}\right)\right\rangle & =\int_{0}^{\mathcal{L}} d z \int_{0}^{\mathcal{L}} d z^{\prime}\left\langle\Delta\left(\zeta_{k}, z\right) \Delta^{*}\left(\zeta_{j}, z^{\prime}\right)\right\rangle \\
& =\int_{0}^{\mathcal{L}} d z \int_{0}^{\mathcal{L}} d z^{\prime} 2 D C\left(z ; \zeta_{k}, \zeta_{j}\right) \delta\left(z-z^{\prime}\right)
\end{aligned}
$$

where we have used Equation (47), and eventually

$$
\left\langle N\left(\zeta_{k}\right) N^{*}\left(\zeta_{j}\right)\right\rangle=2 D \int_{0}^{\mathcal{L}} C\left(z ; \zeta_{k}, \zeta_{j}\right) d z .
$$

Similarly, because of Equation (49), it is

$$
\begin{aligned}
\left\langle N\left(\zeta_{k}\right) N\left(\zeta_{j}\right)\right\rangle & =\int_{0}^{\mathcal{L}} d z \int_{0}^{\mathcal{L}} d z^{\prime}\left\langle\Delta\left(\zeta_{k}, z\right) \Delta\left(\zeta_{j}, z^{\prime}\right)\right\rangle \\
& =\int_{0}^{\mathcal{L}} d z \int_{0}^{\mathcal{L}} d z^{\prime} 2 D E\left(z ; \zeta_{k}, \zeta_{j}\right) \delta\left(z-z^{\prime}\right)
\end{aligned}
$$

or

$$
\left\langle N\left(\zeta_{k}\right) N\left(\zeta_{j}\right)\right\rangle=2 D \int_{0}^{\mathcal{L}} E\left(z ; \zeta_{k}, \zeta_{j}\right) d z .
$$

Both the autocorrelations can be therefore computed with the knowledge of Jost coefficients and Jost functions in the unperturbed case. 


\section{Prati, L. Barletti}

\subsection{The one-soliton case.}

In this section we test our method for the simplest case to treat, that is the one-soliton case, where $N=1$ and the Jost coefficient $b$ is null for real eigenvalues $\xi$. The only discrete eigenvalue in the upper half complex plane is

$$
\zeta_{1}=\alpha_{1}+i \beta_{1} \quad \text { with } \beta_{1}>0 .
$$

In this case, the explicit expression for the Jost functions are known and quite simple [32]. In particular, it is

$$
\begin{aligned}
& \psi_{1}\left(z, t ; \zeta_{1}\right)=\mathrm{e}^{i \zeta_{1} t} \frac{d^{*}}{1+|d|^{2}}, \\
& \psi_{2}\left(z, t ; \zeta_{1}\right)=\mathrm{e}^{i \zeta_{1} t} \frac{1}{1+|d|^{2}},
\end{aligned}
$$

where

$$
d:=-\frac{i C_{1}}{2 \beta_{1}} \mathrm{e}^{2 i \zeta_{1} t}
$$

Autocorrelations for $\Delta$ become (see Equations from (47) to (50)):

$$
\left\langle\Delta\left(\zeta_{1}, z\right) \Delta^{*}\left(\zeta_{1}, z^{\prime}\right)\right\rangle=2 D C\left(z ; \zeta_{1}, \zeta_{1}\right) \delta\left(z-z^{\prime}\right)
$$

and

$$
\left\langle\Delta\left(\zeta_{1}, z\right) \Delta\left(\zeta_{1}, z^{\prime}\right)\right\rangle=2 D E\left(z ; \zeta_{1}, \zeta_{1}\right) \delta\left(z-z^{\prime}\right)
$$

with

$$
C\left(z ; \zeta_{1}, \zeta_{1}\right):=\left|C_{1}(z)\right|^{2} \int_{-\infty}^{+\infty}\left[\left|\psi_{2}\left(z, t ; \zeta_{1}\right)\right|^{4}+\left|\psi_{1}\left(z, t ; \zeta_{1}\right)\right|^{4}\right] d t
$$

and

$$
E\left(z ; \zeta_{1}, \zeta_{1}\right):=C_{k}^{2}(z) \int_{-\infty}^{+\infty} 2 \psi_{2}^{2}\left(z, t ; \zeta_{1}\right) \psi_{1}^{2}\left(z, t ; \zeta_{1}\right) d t
$$

As a consequence, Equation (59) becomes

$$
\begin{aligned}
\left\langle N\left(\zeta_{1}\right) N^{*}\left(\zeta_{1}\right)\right\rangle & =2 D \int_{0}^{\mathcal{L}} C\left(z ; \zeta_{1}, \zeta_{1}\right) d z \\
& =2 D \int_{0}^{\mathcal{L}}\left|C_{1}(z)\right|^{2} \int_{-\infty}^{+\infty}\left[\left|\psi_{2}\left(z, t ; \zeta_{1}\right)\right|^{4}+\left|\psi_{1}\left(z, t ; \zeta_{1}\right)\right|^{4}\right] d t d z \\
& =2 D \int_{0}^{\mathcal{L}}\left|C_{1}(z)\right|^{2} \frac{2 \beta_{1}}{3\left|C_{1}(z)\right|^{2}} d z
\end{aligned}
$$




\section{Discrete eigenvalues for the Stochastic Nonlinear Schrödinger Equation}

and therefore

$$
\left\langle N\left(\zeta_{1}\right) N^{*}\left(\zeta_{1}\right)\right\rangle=\frac{4}{3} D \mathcal{L} \beta_{1} .
$$

Similarly, one obtains

$$
\begin{aligned}
\left\langle N\left(\zeta_{1}\right) N\left(\zeta_{1}\right)\right\rangle & =2 D \int_{0}^{\mathcal{L}} E\left(z ; \zeta_{1}, \zeta_{1}\right) d z \\
& =2 D \int_{0}^{\mathcal{L}} C_{1}^{2}(z) \int_{-\infty}^{+\infty} 2 \psi_{1}^{2}\left(z, t ; \zeta_{1}\right) \psi_{2}^{2}\left(z, t ; \zeta_{1}\right) d t d z \\
& =2 D \int_{0}^{\mathcal{L}} C_{1}^{2}(z)\left[-\frac{\beta_{1}}{3 C_{1}^{2}(z)}\right] d z
\end{aligned}
$$

hence

$$
\left\langle N\left(\zeta_{1}\right) N\left(\zeta_{1}\right)\right\rangle=-\frac{2}{3} D \mathcal{L} \beta_{1} .
$$

The linear growth with the length $\mathcal{L}$ of the fiber is consistent with the analogous linear growth proven in [8] in the case of continuous spectrum. Furthermore, notice that $\left\langle N\left(\zeta_{1}\right) N\left(\zeta_{1}\right)\right\rangle$ is real and negative, therefore the real and the imaginary parts of $N\left(\zeta_{1}\right)$ are uncorrelated and the variance of the imaginary part is greater than that of the real one, see also [25].

These results correspond to the existing literature on the one-soliton case, see for example Chapter 5 of [45]. However our method can be in principle applied to arbitrary multiple soliton case, which will be the subject of our future work.

\section{Conclusions.}

The existing networks and infrastructure for telecommunication services are becoming inadequate because the demand has become increasingly urgent over the years. In the late Seventies, the fiber-optic communications were introduced leading to great technological progress that allowed to exponentially increase the data traffic. The Nonlinear Schrödinger Equation (NLSE) well models the evolution of the complex envelope of signals propagating in optical fibers, and its stochastic version (1) is necessary to account for the unavoidable presence of noise along the fiber. The strong distortion of the optical signal, due to the combined dispersive effects and nonlinear mixing of signal and noise, makes it very difficult to decode the information at the receiver.

The new paradigm of eigenvalue communication, based on the Nonlinear Fourier Transform, is aimed to exploit the mathematical integrability of 


\section{Prati, L. Barletti}

NLSE in order to take advantage from nonlinearity, rather than avoiding it. However, the noise breaks down the perfect integrability of he deterministic NLSE, and this requires an accurate study of the effects of a noisy data on the NFT. In this paper we have integrated the existing studies on the effect of noise on the continuous part of the nonlinear spectrum with an analogous study on the discrete part. Assuming the signal-to-noise ratio to be high, we have used a perturbative approach to obtain, at first order, explicit expressions for the propagated statistics of the discrete eigenvalues along the fiber. Finally, we have tested our results in the mono-solitonic case, that is the case of a single eigenvalue in the upper half complex plane.

\section{Acknowledgements.}

We acknowledge support from Ente Cassa di Risparmio di Firenze, project NOSTRUM - Nonlinear Spectrum Modulation, ref. 2015.0906.

\section{REFERENCES}

1. G. P. Agrawal, Nonlinear Fiber Optics. Academic Press, fifth ed., 2012.

2. M. Secondini and E. Forestieri, The nonlinear Schrödinger equation in fiber-optic systems, Rivista di Matematica della Università di Parma, vol. 8, pp. 69-98, 2008.

3. J. G. Proakis, Digital Communications. McGraw-Hill, 1983.

4. A. Papoulis, Probability, Random Variables, and Stochastic Processes. McGraw-Hill, 1984.

5. A. de Bouard and A. Debussche, The stochastic nonlinear Schrödinger equation in $\mathrm{H}^{1}$, Stochastic Analysis and Applications, vol. 21, no. 1, pp. 97-126, 2003.

6. M. I. Yousefi and F. R. Kschischang, Information transmission using the nonlinear Fourier transform, Part I: Mathematical tools, IEEE Transactions on Information Theory, vol. 60, no. 7, pp. 4312-28, 2014.

7. S. Civelli, L. Barletti, and M. Secondini, Numerical methods for the inverse nonlinear Fourier transform, in Tyrrhenian International Workshop on Digital Communications, TIWDC 2015, IEEE, 2015.

8. S. A. Derevyanko, J. E. Prilepsky, and S. K. Turitsyn, Capacity estimates for optical transmission based on the nonlinear Fourier transform, Nature Communications, vol. 7, no. 12710, 2016.

9. L. Barletti and M. Secondini, Signal-noise interaction in nonlinear optical fibers: a hydrodynamic approach, Optics Express, vol. 23, no. 21, pp. 27419-33, 2015.

10. P. P. Mitra and J. B. Stark, Nonlinear limits to the information capacity of optical fibre communications, Nature, vol. 411, pp. 1027-30, 2001. 


\section{Discrete eigenvalues for the Stochastic Nonlinear Schrödinger Equation}

11. R.-J. Essiambre, G. J. Foschini, G. Kramer, and P. J. Winzer, Capacity limits of information transport in fiber-optic networks, Physical Review Letters, vol. 101, no. 16, p. 163901, 2008.

12. A. D. Ellis, J. Zhao, and D. Cotter, Approaching the non-linear shannon limit, Journal of Lightwave Technology, vol. 28, pp. 423-433, 2010.

13. R.-J. Essiambre, G. Kramer, P. J. Winzer, G. J. Foschini, and B. Goebel, Capacity limits of optical fiber networks, Journal of Lightwave Technology, vol. 28, no. 4, pp. 662-701, 2010.

14. D. J. Richardson, Filling the light pipe, Science, vol. 330, pp. 327-328, October 2010.

15. R. I. Killey and C. Behrens, Shannon's theory in nonlinear systems, Journal of Modern Optics, vol. 58, no. 1, pp. 1-10, 2011.

16. G. Busoni and L. Prati, Some remarks on a linearized Schrödinger equation, Rendiconti Lincei - Matematica e Applicazioni, vol. 26, pp. 1-25, 2015.

17. A. Hasegawa and T. Nyu, Eigenvalue communications, Journal of Lightwave Technology, vol. 11, no. 3, pp. 395-399, 1993.

18. M. J. Ablowitz, D. J. Kaup, A. C. Newell, and H. Segur, Nonlinearevolution equations of physical significance, Physical Review Letters, vol. 31, no. 2, pp. 125-127, 1973.

19. M. J. Ablowitz, D. J. Kaup, A. C. Newell, and H. Segur, The inverse scattering transform - Fourier analysis for nonlinear problems, Studies in Applied Mathematichs, vol. LIII, no. 4, pp. 249-315, 1974.

20. M. J. Ablowitz and H. Segur, Solitons and the Inverse Scattering Transform. SIAM Studies in Applied Mathematics, SIAM, 1981.

21. M. J. Ablowitz and P. A. Clarkson, Solitons, Nonlinear Evolution Equations and Inverse Scattering. London Mathematical Society Lecture Note Series, Cambridge University Press, 1991.

22. S. Oda, A. Maruta, and K. Kitayama, All-optical quantization scheme based on fiber nonlinearity, IEEE Photonics Technology Letters, vol. 16, no. 2, pp. 587-589, 2004.

23. E. Meron, M. Shtaif, and M. Feder, On the achievable communication rates of generalized soliton transmission systems, tech. rep., Preprint at https://arxiv.org/pdf/1207.0297v2.pdf, 2012.

24. H. Terauchi and A. Maruta, Eigenvalue modulated optical transmission system based on digital coherent technology, in Proceedings of 18th OptoElectronics and Communications Conference held jointly with 2013 International Conference on Photonics in Switching (OECC/PS), (Kyoto, Japan), pp. 1-2, IEEE, June 30-July 4, 2013.

25. M. I. Yousefi and F. R. Kschischang, Information transmission using the nonlinear Fourier transform, Part III: Spectrum modulation, IEEE 


\section{Prati, L. Barletti}

Transactions on Information Theory, vol. 60, no. 7, pp. 4346-69, 2014.

26. Z. Dong, S. Hari, T. Gui, K. Zhong, M. I. Yousefi, C. Lu, P.-K. A. Wai, F. R. Kschischang, and A. P. T. Lau, Nonlinear frequency division multiplexed transmission based on nft, IEEE Photonics Technology Letters, vol. 27, no. 15, pp. 1621-23, 2015.

27. S. Hari, M. I. Yousefi, and F. R. Kschischang, Multieigenvalue communication, Journal of Lightwave Technology, vol. 34, no. 13, pp. 3110-17, 2016.

28. J. E. Prilepsky, S. A. Derevyanko, and S. K. Turitsyn, Nonlinear spectral management: linearization of the lossless fiber channel, Optics Express, vol. 21, no. 20, pp. 24344-67, 2013.

29. J. E. Prilepsky, S. A. Derevyanko, K. J. Blow, I. Gabitov, and S. K. Turitsyn, Nonlinear inverse synthesis and eigenvalue division multiplexing in optical fiber channels, Physical Review Letters, vol. 113, p. 013901, July 2014.

30. S. T. Le, J. E. Prilepsky, and S. K. Turitsyn, Nonlinear inverse synthesis for high spectral efficiency transmission in optical fibers, Optic Express, vol. 22, no. 22, pp. 26720-41, 2014.

31. S. T. Le, J. E. Prilepsky, and S. K. Turitsyn, Nonlinear inverse synthesis technique for optical links with lumped amplification, Optic Express, vol. 23, no. 7, pp. 8317-28, 2015.

32. D. J. Kaup, A perturbation expansion for the Zakharov-Shabat inverse scattering transform, SIAM Journal on Applied Mathematics, vol. 31, no. 1, pp. 121-133, 1976.

33. V. I. Karpman and E. M. Maslov, A perturbation theory for the Korteweg de-Vries equation, Physics Letters A, vol. 60, no. 4, pp. 307-308, 1977.

34. D. J. Kaup and A. C. Newell, Solitons as particles, oscillators, and in slowly changing media: A singular perturbation theory, Proceedings of the Royal Society of London. Series A, Mathematical and Physical Sciences, vol. 361, no. 1707, pp. 413-446, 1978.

35. V. I. Karpman and V. V. Solov'ev, A perturbational approach to the two-soliton systems, Physica D: Nonlinear Phenomena, vol. 3, no. 3, pp. 487-502, 1981.

36. V. V. Konotop and L. Vázquez, Nonlinear Random Waves. World Scientific, 1994.

37. P. Kazakopoulos and A. L. Moustakas, Nonlinear Schrödinger equation with random Gaussian input: Distribution of inverse scattering data and eigenvalues, Physical Review E, vol. 78, no. 1, 016603, pp. 1-7, 2008.

38. G. Falkovich, I. Kolokolov, V. Lebedev, V. Mezentsev, and S. Turitsyn, Non-Gaussian error probability in optical soliton transmission, Physica 


\section{Discrete eigenvalues for the Stochastic Nonlinear Schrödinger Equation}

D: Nonlinear Phenomena, vol. 195, no. 1-2, pp. 1-28, 2004.

39. N. J. Zabusky and M. D. Kruskal, Interaction of "solitons" in a collisionless plasma and the recurrence of initial states, Physical Review Letters, vol. 15, no. 6, pp. 240-243, 1965.

40. C. S. Gardner, J. M. Greene, M. D. Kruskal, and R. M. Miura, Method for solving the Korteweg-de Vries equation, Physical Review Letters, vol. 19, no. 19, pp. 1095-97, 1967.

41. P. D. Lax, Integrals of nonlinear equations of evolution and solitary waves, Communications on Pure and Applied Mathematics, vol. XXI, pp. 467-490, 1968.

42. V. E. Zacharov and A. B. Shabat, Exact theory of two-dimensional self-focusing and one-dimensional self-modulation of waves in nonlinear media, Soviet Physics JETP, vol. 34, no. 1, pp. 62-69, 1972.

43. S. Wahls and H. V. Poor, Introducing the fast nonlinear Fourier transform, in IEEE International Conference on Acoustics, Speech and Signal Processing, pp. 5780-84, IEEE, 2013.

44. S. Wahls and H. V. Poor, Fast inverse nonlinear Fourier transform for generating multi-solitons in optical fiber, in IEEE International Symposium on Information Theory, pp. 1676-80, IEEE, 2015.

45. E. Iannone, F. Matera, A. Mecozzi, and M. Settembre, Nonlinear $O p$ tical Communication Networks. Wiley Series in Microwave and Optical Engineering, Wiley, 1998. 